\title{
Bayes Estimation of a Two-Parameter Geometric Distribution under Multiply Type II Censoring
}

\author{
J. B. Shah ${ }^{1}$ and M. N. Patel ${ }^{2}$ \\ ${ }^{1}$ Department of Statistics, R. J. Tibrewal Commerce College, Vastrapur, Ahmedabad, Gujarat 380015, India \\ ${ }^{2}$ Department of Statistics, School of Sciences, Gujarat University, Ahmedabad, Gujarat 380009, India
}

Correspondence should be addressed to J. B. Shah, jayesh782@gmail.com

Received 2 December 2010; Accepted 18 March 2011

Academic Editor: Kwai Sang Chin

Copyright (C) 2011 J. B. Shah and M. N. Patel. This is an open access article distributed under the Creative Commons Attribution License, which permits unrestricted use, distribution, and reproduction in any medium, provided the original work is properly cited.

\begin{abstract}
We derive Bayes estimators of reliability and the parameters of a two- parameter geometric distribution under the general entropy loss, minimum expected loss and linex loss, functions for a noninformative as well as beta prior from multiply Type II censored data. We have studied the robustness of the estimators using simulation and we observed that the Bayes estimators of reliability and the parameters of a two-parameter geometric distribution under all the above loss functions appear to be robust with respect to the correct choice of the hyperparameters $a(b)$ and a wrong choice of the prior parameters $b(a)$ of the beta prior.
\end{abstract}

\section{Introduction}

In life testing experiments, a lot of work has been done under the continuous lifetime models. Sometimes it is neither possible nor convenient to measure the life length of an item continuously until its failure. Also failure time data is sometimes discrete either through the grouping of continuous data due to imprecise measurement or because time itself is discrete. In such circumstances one measures the life of a device on a discrete scale and considers the number of successful cycles, trials, or operations before failure. Therefore, the number of successful trials before failure is more pertinent than the time of continuous period. The oneparameter geometric distribution has an important position in discrete lifetime models. The geometric distribution can be used as a discrete failure to investigate the ability of electronic tubes to withstand successive voltage overloads and performance of electric switches, which are repeatedly turned on and off. Many authors like Yaqub and Khan [1], Patel and Gajjar [2], N. W. Patel and M. N. Patel [3] have contributed to the methodology and estimation of the parameter of the geometric distribution.

Here we assume that the lifetime of certain items has a two-parameter geometric distribution with probability mass function (pmf), cumulative distribution function (cdf), and reliability, respectively, as

$$
\begin{gathered}
p(x)=P(X=x)=p q^{x-M}, \quad F_{X}(x)=1-q^{x-M+1}, \\
R_{X}(x)=q^{x-M+1} ; \quad x=M, M+1, M+2, \ldots, \infty ; \\
M \in N=\{1,2,3, \ldots\}, \quad 0<p<1, \quad q=1-p .
\end{gathered}
$$

Here $p$, and $M$ are unknown parameters.

This model is employed in situations where it is believed that death or failure cannot occur before certain cycles $M$, where $M$ is the warranty time or threshold parameter and $M+(q / p)$ is the expected life of the item. One should be cautious when the model includes a threshold parameter $M$, since the data usually does not provide enough information about $M$ and the inclusion of $M$ could cause rather special statistical problems. If the threshold parameter is known, then there is no difficulty. But if it is unknown then it must be estimated. The models with threshold parameter are difficult to fit with maximum likelihood because for such models likelihood may be unbounded and so maximum likelihood does not work.

In life testing experiments, the experimenter may not always be in a position to observe the lifetimes of all the items 
TABLE 1: Generated observations from geometric distributions.

\begin{tabular}{lccccccccccccccc}
\hline$I$ & 1 & 2 & 3 & 4 & 5 & 6 & 7 & 8 & 9 & 10 & 11 & 12 & 13 & 14 & 15 \\
$x_{i}$ & - & - & - & 3 & 3 & 3 & 3 & 3 & 3 & 3 & 3 & 3 & 3 & 4 & 4 \\
$i$ & 16 & 17 & 18 & 19 & 20 & 21 & 22 & 23 & 24 & 25 & 26 & 27 & 28 & 29 & 30 \\
$x_{i}$ & 4 & 4 & 4 & - & - & - & 6 & 6 & 6 & 6 & 6 & 6 & - & - & - \\
\hline
\end{tabular}

TABLE 2: The values of bayes estimators of $M, p$, and $R\left(t_{0}\right)$ at $t_{0}=6$.

\begin{tabular}{|c|c|c|c|c|c|}
\hline Prior & Loss function & Shape parameter & $\widehat{M}$ & $\hat{p}$ & $\widehat{R}\left(t_{0}\right)$ \\
\hline \multirow{13}{*}{ Informative } & MELF & - & 2 & 0.3629 & 0.1184 \\
\hline & GELF & -3 & 5 & 0.3868 & 0.1730 \\
\hline & GELF & -2 & 5 & 0.3829 & 0.1643 \\
\hline & SELF & -1 & 5 & 0.3790 & 0.1555 \\
\hline & GELF & 1 & 3 & 0.3711 & 0.1370 \\
\hline & GELF & 2 & 3 & 0.3669 & 0.1274 \\
\hline & GELF & 3 & 2 & 0.3628 & 0.1175 \\
\hline & LLF & -3 & 7 & 0.3835 & 0.1590 \\
\hline & LLF & -2 & 7 & 0.3820 & 0.1581 \\
\hline & LLF & -1 & 6 & 0.3806 & 0.1568 \\
\hline & LLF & 1 & 3 & 0.3776 & 0.1541 \\
\hline & LLF & 2 & 2 & 0.3761 & 0.1531 \\
\hline & LLF & 3 & 2 & 0.3747 & 0.1530 \\
\hline \multirow{13}{*}{ Diffuse } & MELF & - & 2 & 0.3760 & 0.1028 \\
\hline & GELF & -3 & 5 & 0.4020 & 0.1613 \\
\hline & GELF & -2 & 5 & 0.3979 & 0.1520 \\
\hline & SELF & -1 & 5 & 0.3937 & 0.1425 \\
\hline & GELF & 1 & 3 & 0.3850 & 0.1226 \\
\hline & GELF & 2 & 3 & 0.3805 & 0.1122 \\
\hline & GELF & 3 & 2 & 0.3759 & 0.1016 \\
\hline & LLF & -3 & 7 & 0.3987 & 0.1461 \\
\hline & LLF & -2 & 7 & 0.3970 & 0.1451 \\
\hline & LLF & -1 & 6 & 0.3954 & 0.1439 \\
\hline & LLF & 1 & 3 & 0.3920 & 0.1411 \\
\hline & LLF & 2 & 2 & 0.3904 & 0.1401 \\
\hline & LLF & 3 & 2 & 0.3887 & 0.1397 \\
\hline \multirow{13}{*}{ Jeffreys' } & MELF & - & 2 & 0.3723 & 0.1043 \\
\hline & GELF & -3 & 5 & 0.3992 & 0.1650 \\
\hline & GELF & -2 & 5 & 0.3950 & 0.1554 \\
\hline & SELF & -1 & 5 & 0.3906 & 0.1455 \\
\hline & GELF & 1 & 3 & 0.3816 & 0.1249 \\
\hline & GELF & 2 & 3 & 0.3769 & 0.1141 \\
\hline & GELF & 3 & 2 & 0.3722 & 0.1031 \\
\hline & LLF & -3 & 7 & 0.4014 & 0.1440 \\
\hline & LLF & -2 & 7 & 0.3996 & 0.1429 \\
\hline & LLF & -1 & 6 & 0.3979 & 0.1417 \\
\hline & LLF & 1 & 3 & 0.3946 & 0.1389 \\
\hline & LLF & 2 & 2 & 0.3929 & 0.1379 \\
\hline & LLF & 3 & 2 & 0.3912 & 0.1374 \\
\hline
\end{tabular}

put on test. This may be because of time limitations and/or other restrictions on data collection. In this situations, Type I or Type II censoring schemes are used. Another way to get censored data is to censor first $r$, middle $t$ and largest $s$ observations thus only available ordered observations are $x_{r+1}, x_{r+2}, \ldots, x_{r+k}$ and $x_{r+k+t+1}, x_{r+k+t+2}, \ldots, x_{N-S}$. The scheme is known as the multiply Type II censoring scheme. This censoring scheme is useful in follow-up studies in epidemiology, reliability and endocrinology. Multiply Type II censoring has been considered by many authors like Balakrishnan [4], Fei and Kong [5], Kong and Fei [6] for continuous lifetime models.

In this paper, we have obtained Bayes estimators of parameters $M, p$, and reliability $R$ of the life model defined in (1) when the data available are of the multiply Type II censored. Here we have considered three types of loss functions, namely, general entropy loss function (GELF), minimum expected loss function (MELF), and linex loss function (LLF). A comparison has been made using Bayes risk among the Bayes estimators. Using simulation study robustness of Bayes estimators has been studied.

\section{Likelihood: Prior, Posterior, and Marginal}

For discrete failure mode, the likelihood function under the multiply Type II censoring scheme described in previous section can be written as

$$
\begin{aligned}
L(\theta \mid x)= & \frac{N !}{r ! t ! s !}\left[F_{X}\left(x_{r}\right)\right]^{r}\left[F_{X}\left(x_{r+k+t}\right)-F_{X}\left(x_{r+k}\right)\right]^{t} \\
& \times\left[1-F_{X}\left(x_{N-S}\right)\right]^{S} \coprod_{i=r+1}^{r+k} p\left(x_{i}\right) \coprod_{i=r+k+t+1}^{N-S} p\left(x_{i}\right) .
\end{aligned}
$$

Using (1) and (2), the likelihood becomes proportional to

$$
L(\theta \mid x) \alpha p^{n}\left(1-q^{V_{1}}\right)^{r}\left(1-q^{V_{2}}\right)^{t} q^{V_{3}},
$$

where

$$
\begin{gathered}
n=N-r-t-s, \quad V_{1}=x_{r}-M+1, \\
V_{2}=x_{r+k+t}-x_{r+k}, \\
V_{3}=\sum_{i=r+1}^{r+k} x_{i}+\sum_{i=r+k+t+1}^{N-s} x_{i}+t x_{r+k}+s x_{N-S}+t+s-M(N-r) .
\end{gathered}
$$

Here we consider beta prior density for $p$ of the form

$$
g_{1}(p) \alpha(1-p)^{a-1} p^{b-1}, \quad 0<p<1 .
$$

If the prior information about $p$ is given in terms of the prior mean $\mu$ and variances $\sigma^{2}$, respectively, then the prior parameters can be obtained by solving

$$
\mu=\frac{b}{a+b}, \quad \sigma^{2}=\frac{a b}{(a+b)^{2}(a+b+1)} .
$$


TABLE 3: The Bayes estimators and their risks under SELF and MELF.

\begin{tabular}{|c|c|c|c|c|c|c|c|}
\hline \multirow{2}{*}{$a$} & \multirow{2}{*}{$b$} & \multicolumn{3}{|c|}{ SELF } & \multicolumn{3}{|c|}{ MELF } \\
\hline & & $\widehat{M}_{S}$ & $\hat{p}_{S}$ & $\hat{R}_{S}\left(t_{0}\right)$ & $\widehat{M}_{M}$ & $\hat{p}_{M}$ & $\hat{R}_{M}\left(t_{0}\right)$ \\
\hline \multirow{6}{*}{7} & 1 & 5 & 0.3629 & 0.1720 & 2 & 0.3459 & 0.1325 \\
\hline & 2 & 5 & 0.3711 & 0.1634 & 2 & 0.3545 & 0.1252 \\
\hline & 3 & 5 & 0.3791 & 0.1555 & 2 & 0.3629 & 0.1184 \\
\hline & 5 & 5 & 0.3945 & 0.1409 & 2 & 0.3791 & 0.1061 \\
\hline & 7 & 5 & 0.4091 & 0.1279 & 2 & 0.3945 & 0.0954 \\
\hline & 9 & 5 & 0.4231 & 0.1165 & 2 & 0.4091 & 0.0860 \\
\hline 1 & & 5 & 0.4104 & 0.1277 & 2 & 0.3937 & 0.0909 \\
\hline 3 & & 5 & 0.3994 & 0.1370 & 2 & 0.3829 & 0.0999 \\
\hline 5 & & 5 & 0.3890 & 0.1462 & 2 & 0.3726 & 0.1092 \\
\hline 7 & 3 & 5 & 0.3791 & 0.1555 & 2 & 0.3629 & 0.1184 \\
\hline 8 & & 5 & 0.3743 & 0.1600 & 2 & 0.3582 & 0.1231 \\
\hline 9 & & 5 & 0.3697 & 0.1646 & 2 & 0.3537 & 0.1277 \\
\hline
\end{tabular}

For $a=b=1$, the above marginal prior reduces to diffuse (or natural) prior given by $g_{1}(p)=1,0<p<1$.

Also the Jeffreys' invariant noninformative prior

$$
g_{1}(p) \alpha(1-p)^{-1} p^{-1 / 2}, \quad 0<p<1,
$$

can be deduced from the above marginal prior with parameters $a=0$ and $b=0.5$.

As in the stand by Broemeling and Tsurumi [7], we suppose the marginal prior distribution of $M$ to be discrete uniform, that is,

$$
g_{2}(M)=\frac{1}{L}, \quad M=1,2,3, \ldots, L(L \in N) .
$$

Using (5) and (8), the joint prior distribution of $p$ and $M$ is

$$
g(p, M) \alpha(1-p)^{a-1} p^{b-1}, \quad 0<p<1 .
$$

The joint posterior density of $p$ and $M$ say $\pi(p, M \mid \underline{x})$, using
(3) and (9), is

$$
\begin{array}{r}
\pi(p, M \mid \underline{x})=\frac{p^{n+b-1}\left(1-q^{V_{1}}\right)^{r}\left(1-q^{V_{2}}\right)^{t} q^{V_{3}+a-1}}{\Psi\left(V_{1}, V_{2}, V_{3}, M, n, a, b\right)}, \\
0<p<1,
\end{array}
$$

where

$$
\begin{aligned}
\Psi\left(V_{1}, V_{2}, V_{3}, M, n, a, b\right)= & \sum_{j_{1}=0}^{r} \sum_{j_{2}=0}^{t} \sum_{M=1}^{L}(-1)^{j_{1}+j_{2}}\left(\begin{array}{l}
r \\
j_{1}
\end{array}\right)\left(\begin{array}{l}
t \\
j_{2}
\end{array}\right) \\
& \times B\left(n+b, V_{1} j_{1}+V_{2} j_{2}+V_{3}+a\right),
\end{aligned}
$$

where $B(m, n)=\int_{0}^{1} x^{m-1}(1-x)^{n-1} d x$.

Marginal posterior distributions of $M$ and $p$ are obtained as

$$
\begin{gathered}
\pi(M \mid \underline{x})=\frac{\sum_{j_{1}=0}^{r} \sum_{j_{2}=0}^{t}(-1)^{j_{1}+j_{2}}\left(\begin{array}{c}
r \\
j_{1}
\end{array}\right)\left(\begin{array}{c}
t \\
j_{2}
\end{array}\right) B\left(n+b, V_{1} j_{1}+V_{2} j_{2}+V_{3}+a\right)}{\Psi\left(V_{1}, V_{2}, V_{3}, M, n, a, b\right)}, \quad M=1,2,3, \ldots, L, \\
\pi(p \mid \underline{x})=\frac{\sum_{j_{1}=0}^{r} \sum_{j_{2}=0}^{t} \sum_{M=1}^{L}(-1)^{j_{1}+j_{2}}\left(\begin{array}{c}
r \\
j_{1}
\end{array}\right)\left(\begin{array}{c}
t \\
j_{2}
\end{array}\right) p^{n+b-1} q^{V_{1} j_{1}+V_{2} j_{2}+V_{3}+a-1}}{\Psi\left(V_{1}, V_{2}, V_{3}, M, n, a, b\right)}, \quad 0<p<1 .
\end{gathered}
$$

\section{Bayes Estimator}

The Bayesian concepts and methods are found useful in Econometrics, Sociology, Engineering, Life Testing and Reliability estimation, Quality Control and others. The Bayes method avoids the possibility of an essentially nonnegative parameter having a negative estimator which could occur when estimation is done using sampling framework. For the given sample and the corresponding likelihood function, Bayes estimator will be unique and there will be no confusion about the choice of the estimator. In estimating certain numbers of parameters in the presence of nuisance parameters (threshold parameters), there would be considerable difficulties in non-Bayesian case whereas in the Bayesian setup inference can be made very simple [8].

The Bayesian method usually requires less sample data to achieve the same quality of inference as compared to the method based on sampling theory. This is an especially 
TABLE 4: The Bayes estimators and their risks under GELF.

\begin{tabular}{|c|c|c|c|c|c|c|c|c|c|c|}
\hline \multirow{2}{*}{$a$} & \multirow{2}{*}{$b$} & \multicolumn{3}{|c|}{$\alpha_{1}=-2$} & \multicolumn{3}{|c|}{$\alpha_{1}=1$} & \multicolumn{3}{|c|}{$\alpha_{1}=2$} \\
\hline & & $\widehat{M}_{G}$ & $\hat{p}_{G}$ & $\hat{R}_{G}\left(t_{0}\right)$ & $\widehat{M}_{G}$ & $\hat{p}_{G}$ & $\hat{R}_{G}\left(t_{0}\right)$ & $\widehat{M}_{G}$ & $\hat{p}_{G}$ & $\hat{R}_{G}\left(t_{0}\right)$ \\
\hline \multirow{6}{*}{7} & 1 & 5 & 0.3670 & 0.1813 & 3 & 0.3545 & 0.1524 & 3 & 0.3501 & 0.1421 \\
\hline & 2 & 5 & 0.3751 & 0.1726 & 3 & 0.3629 & 0.1444 & 3 & 0.3587 & 0.1345 \\
\hline & 3 & 5 & 0.3830 & 0.1643 & 3 & 0.3711 & 0.1370 & 3 & 0.3670 & 0.1274 \\
\hline & 5 & 5 & 0.3982 & 0.1493 & 3 & 0.3869 & 0.1235 & 3 & 0.3830 & 0.1145 \\
\hline & 7 & 5 & 0.4126 & 0.1359 & 3 & 0.4019 & 0.1116 & 3 & 0.3982 & 0.1032 \\
\hline & 9 & 5 & 0.4265 & 0.1239 & 3 & 0.4162 & 0.1011 & 3 & 0.4126 & 0.0932 \\
\hline 1 & & 5 & 0.4144 & 0.1367 & 3 & 0.4022 & 0.1092 & 3 & 0.3979 & 0.0996 \\
\hline 3 & & 5 & 0.4033 & 0.1459 & 3 & 0.3912 & 0.1184 & 3 & 0.3870 & 0.1088 \\
\hline 5 & & 5 & 0.3929 & 0.1552 & 3 & 0.3809 & 0.1277 & 3 & 0.3767 & 0.1181 \\
\hline 7 & 3 & 5 & 0.3830 & 0.1643 & 3 & 0.3711 & 0.1370 & 3 & 0.3670 & 0.1274 \\
\hline 8 & & 5 & 0.3782 & 0.1689 & 3 & 0.3664 & 0.1416 & 3 & 0.3623 & 0.1320 \\
\hline 9 & & 5 & 0.3735 & 0.1734 & 3 & 0.3618 & 0.1462 & 3 & 0.3577 & 0.1367 \\
\hline
\end{tabular}

TABLE 5: The Bayes estimators and their risks under LLF.

\begin{tabular}{|c|c|c|c|c|c|c|c|}
\hline \multirow{2}{*}{$a$} & \multirow{2}{*}{$b$} & \multicolumn{3}{|c|}{$\alpha_{2}=-1$} & \multicolumn{3}{|c|}{$\alpha_{2}=-2$} \\
\hline & & $\widehat{M}_{L}$ & $\hat{p}_{L}$ & $\hat{R}_{L}\left(t_{0}\right)$ & $\widehat{M}_{L}$ & $\hat{p}_{L}$ & $\hat{R}_{L}\left(t_{0}\right)$ \\
\hline \multirow{6}{*}{7} & 1 & 6 & 0.3644 & 0.1736 & 7 & 0.3659 & 0.1749 \\
\hline & 2 & 6 & 0.3726 & 0.1650 & 7 & 0.3741 & 0.1662 \\
\hline & 3 & 6 & 0.3806 & 0.1568 & 7 & 0.3820 & 0.1581 \\
\hline & 5 & 6 & 0.3959 & 0.1421 & 7 & 0.3974 & 0.1431 \\
\hline & 7 & 6 & 0.4106 & 0.1290 & 7 & 0.4120 & 0.1299 \\
\hline & 9 & 6 & 0.4245 & 0.1174 & 7 & 0.4260 & 0.1182 \\
\hline 1 & & 6 & 0.4120 & 0.1289 & 7 & 0.4137 & 0.1300 \\
\hline 3 & & 6 & 0.4009 & 0.1382 & 7 & 0.4026 & 0.1394 \\
\hline 5 & & 6 & 0.3905 & 0.1476 & 7 & 0.3920 & 0.1487 \\
\hline 7 & 3 & 6 & 0.3806 & 0.1568 & 7 & 0.3820 & 0.1581 \\
\hline 8 & & 6 & 0.3758 & 0.1615 & 7 & 0.3772 & 0.1627 \\
\hline 9 & & 6 & 0.3711 & 0.1661 & 7 & 0.3726 & 0.1673 \\
\hline
\end{tabular}

TABLE 6: The Bayes estimators and their risks under LLF.

\begin{tabular}{|c|c|c|c|c|c|c|c|}
\hline \multirow{2}{*}{$a$} & \multirow{2}{*}{$b$} & \multicolumn{3}{|c|}{$\alpha_{2}=1$} & \multicolumn{3}{|c|}{$\alpha_{2}=2$} \\
\hline & & $\widehat{M}_{L}$ & $\hat{p}_{L}$ & $\hat{R}_{L}\left(t_{0}\right)$ & $\widehat{M}_{L}$ & $\hat{p}_{L}$ & $\hat{R}_{L}\left(t_{0}\right)$ \\
\hline \multirow{6}{*}{7} & 1 & 3 & 0.3614 & 0.1704 & 2 & 0.3599 & 0.1694 \\
\hline & 2 & 3 & 0.3696 & 0.1620 & 2 & 0.3681 & 0.1610 \\
\hline & 3 & 3 & 0.3776 & 0.1541 & 2 & 0.3761 & 0.1531 \\
\hline & 5 & 3 & 0.3930 & 0.1397 & 2 & 0.3916 & 0.1388 \\
\hline & 7 & 3 & 0.4072 & 0.1269 & 2 & 0.4062 & 0.1261 \\
\hline & 9 & 3 & 0.4217 & 0.1156 & 2 & 0.4203 & 0.1148 \\
\hline 1 & & 3 & 0.4088 & 0.1266 & 2 & 0.4071 & 0.1256 \\
\hline 3 & & 3 & 0.3978 & 0.1358 & 2 & 0.3962 & 0.1348 \\
\hline 5 & & 3 & 0.3874 & 0.1450 & 2 & 0.3859 & 0.1440 \\
\hline 7 & 3 & 3 & 0.3776 & 0.1541 & 2 & 0.3761 & 0.1531 \\
\hline 8 & & 3 & 0.3729 & 0.1587 & 2 & 0.3714 & 0.1577 \\
\hline 9 & & 3 & 0.3683 & 0.1632 & 2 & 0.3669 & 0.1623 \\
\hline
\end{tabular}


TABLE 7: The Bayes estimators and their risks under SELF and MELF.*

\begin{tabular}{|c|c|c|c|c|c|c|c|}
\hline \multirow{2}{*}{$a$} & \multirow{2}{*}{$b$} & \multicolumn{3}{|c|}{ SELF } & \multicolumn{3}{|c|}{ MELF } \\
\hline & & $\widehat{M}_{S}$ & $\hat{p}_{S}$ & $\hat{R}_{S}\left(t_{0}\right)$ & $\widehat{M}_{M}$ & $\hat{p}_{M}$ & $\hat{R}_{M}\left(t_{0}\right)$ \\
\hline \multirow{12}{*}{7} & \multirow{2}{*}{1} & 4 & 0.2961 & 0.2572 & 2 & 0.2810 & 0.2224 \\
\hline & & 0.07469 & 0.01105 & 0.03011 & 0.11111 & 0.02336 & 0.07138 \\
\hline & \multirow{2}{*}{2} & 4 & 0.3034 & 0.2471 & 2 & 0.2887 & 0.2129 \\
\hline & & 0.07411 & 0.01030 & 0.03207 & 0.11111 & 0.02140 & 0.07604 \\
\hline & \multirow{2}{*}{3} & 4 & 0.3106 & 0.2375 & 2 & 0.2961 & 0.2040 \\
\hline & & 0.08128 & 0.01015 & 0.03591 & 0.11111 & 0.02073 & 0.08321 \\
\hline & \multirow{2}{*}{5} & 4 & 0.3244 & 0.2196 & 2 & 0.3106 & 0.1875 \\
\hline & & 0.07528 & 0.01135 & 0.04931 & 0.11111 & 0.02290 & 0.10346 \\
\hline & \multirow{2}{*}{7} & 4 & 0.3377 & 0.2035 & 2 & 0.3244 & 0.1727 \\
\hline & & 0.08362 & 0.01411 & 0.07050 & 0.11111 & 0.02929 & 0.12949 \\
\hline & \multirow{2}{*}{9} & 4 & 0.3505 & 0.1888 & 2 & 0.3377 & 0.1594 \\
\hline & & 0.07996 & 0.01799 & 0.09969 & 0.11111 & 0.03938 & 0.15932 \\
\hline \multirow{2}{*}{1} & \multirow{8}{*}{3} & 4 & 0.3316 & 0.2125 & 2 & 0.3167 & 0.1781 \\
\hline & & 0.08098 & 0.01433 & 0.07098 & 0.11111 & 0.03006 & 0.13048 \\
\hline \multirow{2}{*}{3} & & 4 & 0.3243 & 0.2209 & 2 & 0.3095 & 0.1868 \\
\hline & & 0.07981 & 0.01246 & 0.05556 & 0.11111 & 0.02558 & 0.11215 \\
\hline \multirow{2}{*}{5} & & 4 & 0.3173 & 0.2293 & 2 & 0.3027 & 0.1954 \\
\hline & & 0.08245 & 0.01108 & 0.04414 & 0.11111 & 0.02253 & 0.09640 \\
\hline \multirow{2}{*}{7} & & 4 & 0.3106 & 0.2375 & 2 & 0.2961 & 0.2040 \\
\hline & & 0.08128 & 0.01015 & 0.03591 & 0.11111 & 0.02073 & 0.08321 \\
\hline \multirow{2}{*}{8} & & 4 & 0.3073 & 0.2416 & 2 & 0.2929 & 0.2083 \\
\hline & & 0.08347 & 0.00985 & 0.03279 & 0.11111 & 0.02024 & 0.07757 \\
\hline \multirow{2}{*}{9} & & 4 & 0.03042 & 0.2456 & 2 & 0.2898 & 0.2125 \\
\hline & & 0.08259 & 0.00967 & 0.03026 & 0.11111 & 0.02000 & 0.07256 \\
\hline
\end{tabular}

${ }^{*}$ In each cell the first value gives Bayes estimate and second its risk.

important consideration in those areas of application where sample data may be either expensive or difficult to obtain, for example as in reliability. Over the years, reliability estimation methods based on sampling theory have been found to be extremely useful for a wide variety of problems. However, there are many instances in which the classical methods have been found to be less satisfactory. As reliability testing is directly related to cost and time, the use of sampling theory would be extremely limited. Sampling theory methods are especially inappropriate for reliability analysis based on scarce data.

Most of the engineering designs are evolutionary rather than revolutionary processes in which current equipment is modified to suit new requirements. In such instances it would appear that the known facts regarding reliability of the current hardware could possibly be utilized in an attempt to improve the quality of the reliability estimates of the new design. The sampling theory methods are inappropriate for incorporating such related information.

In reliability estimation, the Bayesian analysis has been found useful due to two facts: one is the increased quality of the inferences; provided the prior information accurately reflects the true variation in the parameter(s); the other is the reduction in testing requirements (like test time, sample size, etc.) that often occur in Bayesian reliability demonstration test programs. The Bayesian method produces believable results which go a long way in convincing the engineer of the utility of Bayesian methods.

\section{Bayes Estimators under Minimum Expected Loss Function (MELF)}

In Bayesian analysis, widely used loss function is a quadratic loss function given by

$$
L_{1}(\theta, d)=w(d-\theta)^{2} .
$$

If $w=1$, it reduces to squared error loss function (SELF) and for $w=\theta^{-2}$, it becomes

$$
L_{1}(\theta, d)=\theta^{-2}(d-\theta)^{2}
$$

known as MELF introduced by Rao Tummala and Sathe [9] in their study.

The Bayes estimators of $\theta$ under MELF are given by

$$
\hat{\theta}_{M}=\frac{E\left(\theta^{-1}\right)}{E\left(\theta^{-2}\right)} .
$$

For the threshold parameter $M$, which is a positive integer quantity $M=1,2,3, \ldots, L(L \in N)$, the loss function is defined as $L_{1}\left(M, \widehat{M}_{M}\right)=\left(M-\widehat{M}_{M}\right)^{2}$, where 
TABLE 8: The bayes estimators and their risks under GELF.*

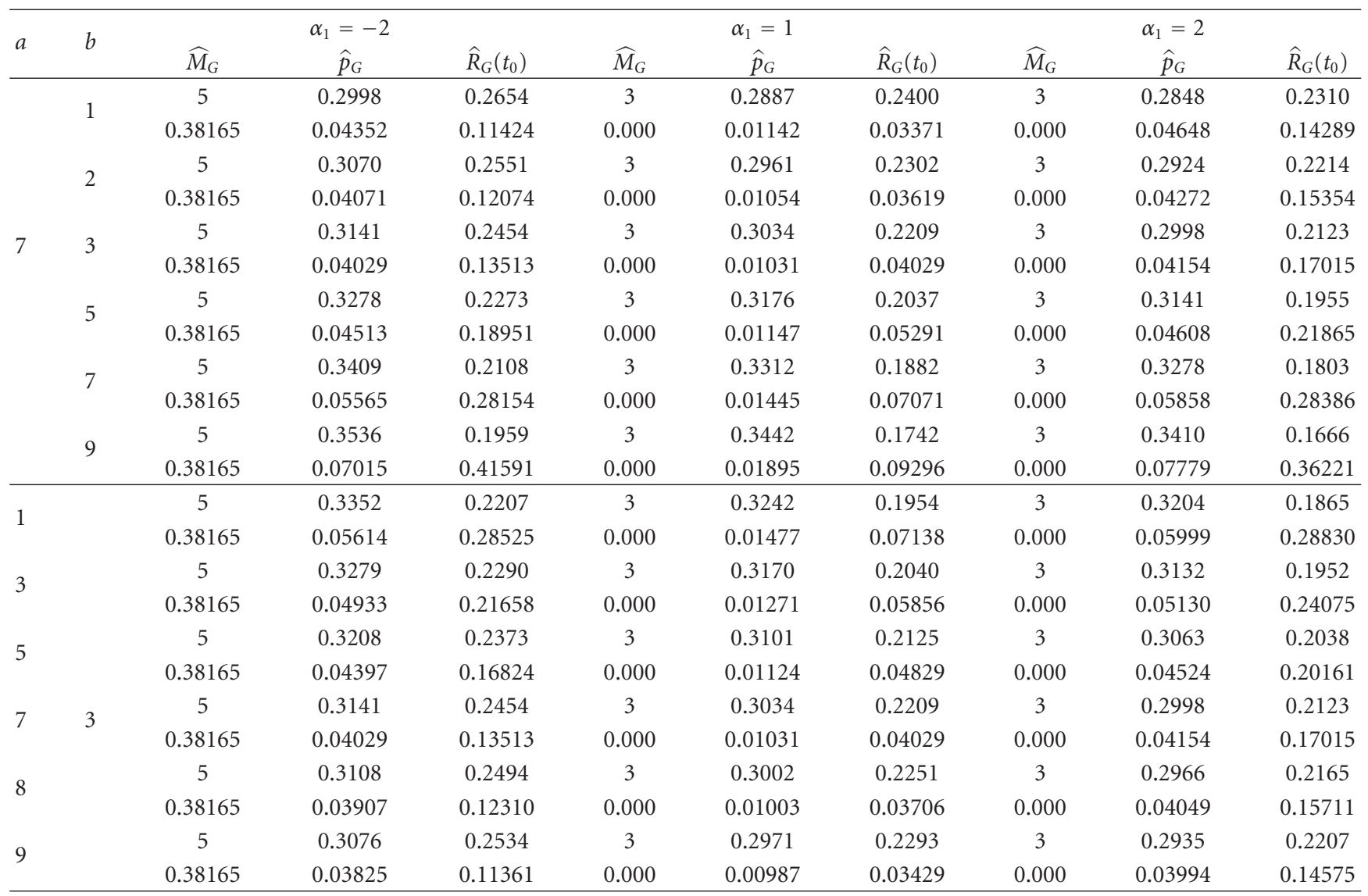

* In each cell the first value gives Bayes estimate and second its risk.

$\widehat{M}_{M}=1,2,3, \ldots, L(L \in N)$ is the smallest integer greater than the analytical solution. are

The Bayes estimators of $M, p$ and $R\left(t_{0}\right)$ under MELF

$$
\begin{gathered}
\widehat{M}_{M}=\frac{\sum_{j_{1}=0}^{r} \sum_{j_{2}=0}^{t} \sum_{M=1}^{L} M^{-1}(-1)^{j_{1}+j_{2}}\left(\begin{array}{c}
r \\
j_{1}
\end{array}\right)\left(\begin{array}{c}
t \\
j_{2}
\end{array}\right) B\left(n+b, V_{1} j_{1}+V_{2} j_{2}+V_{3}+a\right)}{\sum_{j_{1}=0}^{r} \sum_{j_{2}=0}^{t} \sum_{M=1}^{L} M^{-2}(-1)^{j_{1}+j_{2}}\left(\begin{array}{c}
r \\
j_{1}
\end{array}\right)\left(\begin{array}{c}
t \\
j_{2}
\end{array}\right) B\left(n+b, V_{1} j_{1}+V_{2} j_{2}+V_{3}+a\right)}, \\
\hat{p}_{M}=\frac{\Psi\left(V_{1}, V_{2}, V_{3}, M, n, a, b-1\right)}{\Psi\left(V_{1}, V_{2}, V_{3}, M, n, a, b-2\right)}, \\
\hat{R}_{M}\left(t_{0}\right)=\frac{\Psi\left(V_{1}, V_{2}, V_{3}, M, n, a-\left(t_{0}-M+1\right), b\right)}{\Psi\left(V_{1}, V_{2}, V_{3}, M, n, a-2\left(t_{0}-M+1\right), b\right)} .
\end{gathered}
$$

\section{Bayes Estimators under General Entropy Loss Function (GELF)}

Sometimes, the use of symmetric loss function, namely, SELF, was found to be inappropriate in a situation where an overestimation of the reliability function is usually much more serious than an underestimation. Here we consider the asymmetric loss function, namely, GELF, proposed by Calabria and Pulcini [10], is

$$
L_{2}(\theta, d)=\left(\frac{d}{\theta}\right)^{\alpha_{1}}-\alpha_{1} \ln \left(\frac{d}{\theta}\right)-1, \quad \alpha_{1} \neq 0,
$$

whereas for the threshold parameter $M$, the loss function is defined as

$$
L_{2}\left(M, \widehat{M}_{G}\right)=\left(\frac{\widehat{M}_{G}}{M}\right)^{\alpha_{1}}-\alpha_{1} \ln \left(\frac{\widehat{M}_{G}}{M}\right)-1,
$$

where $\alpha_{1} \neq 0, M=1,2,3, \ldots, L$, and $\widehat{M}_{G}=1,2,3, \ldots, L(L \in$ $N)$. Here $\widehat{M}_{G}$ is the smallest integer greater than the analytical solution. The sign of the shape parameter $\alpha_{1}$ reflects the deviation of the asymmetry, $\alpha_{1}>0$ if overestimation is more serious than underestimation, and vice versa. The magnitude 
TABLE 9: The bayes estimators and their risks under LLF.*

\begin{tabular}{|c|c|c|c|c|c|c|c|}
\hline \multirow{2}{*}{$a$} & \multirow{2}{*}{$b$} & \multicolumn{3}{|c|}{$\alpha_{2}=-1$} & \multicolumn{3}{|c|}{$\alpha_{2}=-2$} \\
\hline & & $\widehat{M}_{L}$ & $\hat{p}_{L}$ & $\hat{R}_{L}\left(t_{0}\right)$ & $\widehat{M}_{L}$ & $\hat{p}_{L}$ & $\hat{R}_{L}\left(t_{0}\right)$ \\
\hline \multirow{12}{*}{7} & \multirow{2}{*}{1} & 6 & 0.2972 & 0.2591 & 7 & 0.2983 & 0.2598 \\
\hline & & 2.0498 & 0.00094 & 0.00185 & 7.0003 & 0.00377 & 0.00717 \\
\hline & \multirow{2}{*}{2} & 6 & 0.3045 & 0.2489 & 7 & 0.3056 & 0.2497 \\
\hline & & 2.0498 & 0.00096 & 0.00167 & 7.0003 & 0.00387 & 0.00653 \\
\hline & \multirow{2}{*}{3} & 6 & 0.3117 & 0.2392 & 7 & 0.3128 & 0.2400 \\
\hline & & 2.0498 & 0.00104 & 0.00016 & 7.0003 & 0.00417 & 0.00628 \\
\hline & \multirow{2}{*}{5} & 6 & 0.3255 & 0.2212 & 7 & 0.3267 & 0.2221 \\
\hline & & 2.0498 & 0.00132 & 0.00170 & 7.0003 & 0.00530 & 0.00675 \\
\hline & \multirow{2}{*}{7} & 6 & 0.3389 & 0.2049 & 7 & 0.3400 & 0.2058 \\
\hline & & 2.0498 & 0.00177 & 0.00206 & 7.0003 & 0.00706 & 0.00828 \\
\hline & \multirow{2}{*}{9} & 6 & 0.3516 & 0.1901 & 7 & 0.3527 & 0.1909 \\
\hline & & 2.0498 & 0.00236 & 0.00262 & 7.0003 & 0.00935 & 0.01061 \\
\hline \multirow{2}{*}{1} & \multirow{8}{*}{3} & 6 & 0.3329 & 0.2142 & 7 & 0.3341 & 0.2151 \\
\hline & & 2.0498 & 0.00177 & 0.00210 & 7.0003 & 0.00705 & 0.00842 \\
\hline \multirow{2}{*}{3} & & 6 & 0.3255 & 0.2226 & 7 & 0.3267 & 0.2235 \\
\hline & & 2.0498 & 0.00146 & 0.00186 & 7.0003 & 0.00584 & 0.00741 \\
\hline \multirow{2}{*}{5} & & 6 & 0.3184 & 0.2309 & 7 & 0.3196 & 0.2318 \\
\hline & & 2.0498 & 0.00122 & 0.00170 & 7.0003 & 0.00489 & 0.00670 \\
\hline \multirow{2}{*}{7} & & 6 & 0.3117 & 0.2392 & 7 & 0.3128 & 0.2400 \\
\hline & & 2.0498 & 0.00104 & 0.00016 & 7.0003 & 0.00417 & 0.00628 \\
\hline \multirow{2}{*}{8} & & 6 & 0.3084 & 0.2432 & 7 & 0.3095 & 0.2441 \\
\hline & & 2.0498 & 0.00097 & 0.00157 & 7.0003 & 0.00389 & 0.00617 \\
\hline \multirow{2}{*}{9} & & 6 & 0.3052 & 0.2473 & 7 & 0.3063 & 0.2481 \\
\hline & & 2.0498 & 0.00091 & 0.00156 & 7.0003 & 0.00366 & 0.00612 \\
\hline
\end{tabular}

* In each cell the first value gives Bayes estimate and second its risk.

of $\alpha_{1}$ reflects the degree of asymmetry. The Bayes estimator of $\theta$ under the GELF given in (17) is given by

$$
\hat{\theta}_{G}=\left[E_{\pi}\left(\theta^{-\alpha_{1}}\right)\right]^{-1 / \alpha_{1}} .
$$

$$
\widehat{M}_{G}=\left[\frac{\sum_{j_{1}=0}^{r} \sum_{j_{2}=0}^{t} \sum_{M=1}^{L} M^{-\alpha_{1}}(-1)^{j_{1}+j_{2}}\left(\begin{array}{c}
r \\
j_{1}
\end{array}\right)\left(\begin{array}{c}
t \\
j_{2}
\end{array}\right) B\left(n+b, V_{1} j_{1}+V_{2} j_{2}+V_{3}+a\right)}{\Psi\left(V_{1}, V_{2}, V_{3}, M, n, a, b\right)}\right]^{-1 / \alpha_{1}},
$$

$$
\hat{p}_{G}=\left[\frac{\Psi\left(V_{1}, V_{2}, V_{3}, M, n, a, b-\alpha_{1}\right)}{\Psi\left(V_{1}, V_{2}, V_{3}, M, n, a, b\right)}\right]^{-1 / \alpha_{1}}
$$

$\hat{R}_{G}\left(t_{0}\right)=\left[\frac{\Psi\left(V_{1}, V_{2}, V_{3}, M, n, a-\alpha_{1}\left(t_{0}-M+1\right), b\right)}{\Psi\left(V_{1}, V_{2}, V_{3}, M, n, a, b\right)}\right]^{-1 / \alpha_{1}}$.

The Bayes estimators of $M, p$, and $R\left(t_{0}\right)$ under SELF can be obtained by substituting $\alpha_{1}=-1$ in the results (20) to (22), respectively.
The Bayes estimators of $M, p$, and $R\left(t_{0}\right)$ under GELF are, respectively,

\section{Bayes Estimators under Linex Loss Function (LLF)}

Another asymmetric loss function given by Varian [11], known as LLF, is defined as

$$
L_{3}(\theta, d)=\exp \left[\alpha_{2}(d-\theta)\right]-\alpha_{2}(d-\theta)-1 \quad\left(\alpha_{2} \neq o\right),
$$


whereas for the threshold parameter $M$, the loss function is defined as

$$
\begin{aligned}
L_{3}\left(M, \widehat{M}_{L}\right)= & \exp \left[\alpha_{2}\left(\widehat{M}_{L}-M\right)\right] \\
& -\alpha_{2}\left(\widehat{M}_{L}-M\right)-1 \quad\left(\alpha_{2} \neq 0\right),
\end{aligned}
$$

where $M=1,2,3, \ldots, L$ and $\widehat{M}_{G}=1,2,3, \ldots, L(L \in N)$. Here $\widehat{M}_{L}$ is the smallest integer greater than the analytical solution. The sign and magnitude of the shape parameter $\alpha_{2}$ reflect the deviation and degree of asymmetry, respectively. The Bayes estimator of $\theta$ under LLF given in (23) is given by

$$
\hat{\theta}_{L}=\frac{-1}{\alpha_{2}} \ln E_{\pi}\left[\exp \left(-\alpha_{2} \theta\right)\right] .
$$

Using marginal posterior distributions (12) in (25) and formula 3.478 (3), page 364 of Gradshteyn and Ryzhik [12], the Bayes estimators of $M, p$, and $R\left(t_{0}\right)$, respectively, can be obtained as

$$
\begin{aligned}
& \widehat{M}_{L}=\frac{-1}{\alpha_{2}} \ln \left[\frac{\sum_{j_{1}=0}^{r} \sum_{j_{2}=0}^{t} \sum_{M=1}^{L} e^{-\alpha_{2} M}(-1)^{j_{1}+j_{2}}\left(\begin{array}{c}
r \\
j_{1}
\end{array}\right)\left(\begin{array}{c}
t \\
j_{2}
\end{array}\right) B\left(n+b, V_{1} j_{1}+V_{2} j_{2}+V_{3}+a\right)}{\Psi\left(V_{1}, V_{2}, V_{3}, M, n, a, b\right)}\right],
\end{aligned}
$$

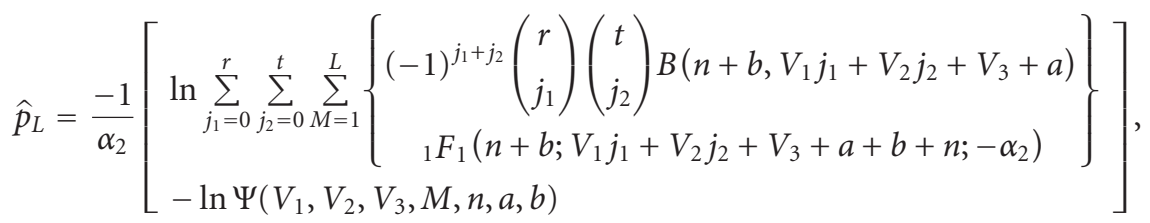

$$
\begin{aligned}
& \hat{R}_{L}\left(t_{0}\right)=\frac{-1}{\alpha_{2}} \ln \sum_{j_{1}=0}^{r} \sum_{j_{2}=0}^{t} \sum_{M=1}^{L}\left\{\left\{\begin{array}{r}
(-1)^{j_{1}+j_{2}}\left(\begin{array}{l}
r \\
j_{1}
\end{array}\right)\left(\begin{array}{l}
t \\
j_{2}
\end{array}\right) B\left(n+b, V_{1} j_{1}+V_{2} j_{2}+V_{3}+a\right) \\
\left(t_{0}-M+1\right) F_{\left(t_{0}-M+1\right)}\left(\frac{V_{1} j_{1}+V_{2} j_{2}+V_{3}+a}{t_{0}-M+1}, \frac{V_{1} j_{1}+V_{2} j_{2}+V_{3}+a+1}{t_{0}-M+1}, \ldots,\right. \\
\frac{V_{1} j_{1}+V_{2} j_{2}+V_{3}+a+t_{0}-M}{t_{0}-M+1} ; \frac{V_{1} j_{1}+V_{2} j_{2}+V_{3}+n+a+b}{t_{0}-M+1}, \\
-\ln \Psi\left(V_{1}, V_{2}, V_{3}, M, n, a, b\right)
\end{array}\right],\right.
\end{aligned}
$$

where ${ }_{p} F_{q}\left(\alpha_{1}, \alpha_{2}, \ldots, \alpha_{p} ; \beta_{1}, \beta_{2}, \ldots, \beta_{q} ; z\right)$ is called a generalized hypergeometric series which is defined as follows (see Gradshteyn and Ryzhik (see [12, page 1000, formula 9.14 (1)]:

$$
\begin{gathered}
{ }_{p} F_{q}\left(\alpha_{1}, \alpha_{2}, \ldots, \alpha_{p} ; \beta_{1}, \beta_{2}, \ldots, \beta_{q} ; z\right) \\
=\sum_{k=0}^{\infty} \frac{\left(\alpha_{1}\right)_{k}\left(\alpha_{2}\right)_{k} \cdots\left(\alpha_{p}\right)_{k}}{\left(\beta_{1}\right)_{k}\left(\beta_{2}\right)_{k} \cdots\left(\beta_{q}\right)_{k}} \frac{(z)}{k !}
\end{gathered}
$$

where $\left(\gamma_{i}\right)_{k}=\left(\gamma_{i}\right)\left(\gamma_{i}+1\right)\left(\gamma_{i}+2\right) \cdots\left(\gamma_{i}+k-1\right)$.

The underconsidered multiply Type II censoring scheme reduces to right Type II, left Type II, and doubly Type II censoring schemes when $r=0$ and $t=0, t=0$, and $s=0$ and $t=0$, respectively.

\section{Numerical Example}

We have generated 30 random observations with $M=3, L=$ $7, p=0.3$ from the distribution defined in (1) having reliability $R\left(t_{0}\right)=0.2401$ at $t_{0}=6$. Suppose that information about first 3 , middle 3 , and largest 3 observations are censored, then the only available observations are given in Table 1 .

In Table $1, N=30, r=t=s=3, k=15$. Prior parameters $a$ and $b$ themselves were random observations from beta distribution with means $\mu=0.3$ and variance $\sigma^{2}=0.0233$, resulting in $a=7$ and $b=3$. We have calculated Bayes estimators of $M, p$ and $R\left(t_{0}\right)$ at $t_{0}=6$ under MELF, SELF, GELF, and LLF. For a comparative purpose, point estimates under the diffuse prior $(a=1, b=1)$ and Jeffreys' prior ( $a=0, b=0.5$ ) have also been calculated.

The results are shown in Table 2.

From Table 2, we observed that the Bayes estimator of $M$ moves away from the true value of $M$ under $\left|\alpha_{1}\right| \geq 2$ for GELF and $\left|\alpha_{2}\right| \geq 2$ for LLF, but the Bayes estimators of the other parameters are not affected, so much, under these choices. So, in subsequent Sections 8 and 9, we have considered the values of $\alpha_{1}$ only $-2,-1,1$, and 2 whereas for $\alpha_{2}$ only $-2,-1,1$, and 2 for the comparison. 
TABLE 10: The bayes estimators and their risks under LLF.

\begin{tabular}{|c|c|c|c|c|c|c|c|}
\hline \multirow{2}{*}{$a$} & \multirow{2}{*}{$b$} & \multicolumn{3}{|c|}{$\alpha_{2}=1$} & \multicolumn{3}{|c|}{$\alpha_{2}=2$} \\
\hline & & $\widehat{M}_{L}$ & $\hat{p}_{L}$ & $\hat{R}_{L}\left(t_{0}\right)$ & $\widehat{M}_{L}$ & $\hat{p}_{L}$ & $\hat{R}_{L}\left(t_{0}\right)$ \\
\hline \multirow{12}{*}{7} & \multirow{2}{*}{1} & 3 & 0.2950 & 0.2555 & 2 & 0.2940 & 0.2572 \\
\hline & & 0.000 & 0.00093 & 0.00186 & 1.1353 & 0.00369 & 0.00845 \\
\hline & \multirow{2}{*}{2} & 3 & 0.3023 & 0.2455 & 2 & 0.3013 & 0.2467 \\
\hline & & 0.000 & 0.00095 & 0.00168 & 1.1353 & 0.00378 & 0.00742 \\
\hline & \multirow{2}{*}{3} & 3 & 0.3095 & 0.2359 & 2 & 0.3084 & 0.2368 \\
\hline & & 0.000 & 0.00102 & 0.00160 & 1.1353 & 0.00407 & 0.00690 \\
\hline & \multirow{2}{*}{5} & 3 & 0.3233 & 0.2182 & 2 & 0.3223 & 0.2168 \\
\hline & & 0.000 & 0.00131 & 0.00169 & 1.1353 & 0.00524 & 0.00698 \\
\hline & \multirow{2}{*}{7} & 3 & 0.3367 & 0.2022 & 2 & 0.3356 & 0.2022 \\
\hline & & 0.000 & 0.00178 & 0.00204 & 1.1353 & 0.00711 & 0.00817 \\
\hline & \multirow{2}{*}{9} & 3 & 0.3494 & 0.1876 & 2 & 0.3483 & 0.1874 \\
\hline & & 0.000 & 0.00239 & 0.00256 & 1.1353 & 0.00963 & 0.01014 \\
\hline \multirow{2}{*}{1} & \multirow{8}{*}{3} & 3 & 0.3304 & 0.2110 & 2 & 0.3292 & 0.2112 \\
\hline & & 0.000 & 0.00177 & 0.00208 & 1.1353 & 0.00711 & 0.00850 \\
\hline \multirow{2}{*}{3} & & 3 & 0.3231 & 0.2194 & 2 & 0.3220 & 0.2198 \\
\hline & & 0.000 & 0.00145 & 0.00185 & 1.1353 & 0.00580 & 0.00769 \\
\hline \multirow{2}{*}{5} & & 3 & 0.3162 & 0.2277 & 2 & 0.3150 & 0.2283 \\
\hline & & 0.000 & 0.00121 & 0.00169 & 1.1353 & 0.00480 & 0.00716 \\
\hline \multirow{2}{*}{7} & & 3 & 0.3095 & 0.2359 & 2 & 0.3084 & 0.2368 \\
\hline & & 0.000 & 0.00102 & 0.00160 & 1.1353 & 0.00407 & 0.00690 \\
\hline \multirow{2}{*}{8} & & 3 & 0.3063 & 0.2400 & 2 & 0.3052 & 0.2410 \\
\hline & & 0.000 & 0.00096 & 0.00158 & 1.1353 & 0.00380 & 0.00688 \\
\hline \multirow{2}{*}{9} & & 3 & 0.3031 & 0.2440 & 2 & 0.2950 & 0.2553 \\
\hline & & 0.000 & 0.00090 & 0.00157 & 1.1353 & 0.00347 & 0.00780 \\
\hline
\end{tabular}

* In each cell the first value gives Bayes estimate and second its risk.

\section{Sensitivity of Bayes Estimators}

In this section, we have studied the sensitivity of the Bayes estimators with respect to changes in the parameters of the beta prior. We have computed Bayes estimators of $M, p$ and $R\left(t_{0}\right)$ at $t_{0}=6$ in case of MELF, SELF, GELF, and LLF considering different sets of values of $(a, b)$ for the data given in Table 1. The results are shown in Tables 3-6.

Following Calabria and Pulcini [13], we also assume the prior information to be correct if the true value of $p$ is close to prior mean $\mu$ and is assumed to be wrong if $p$ is far from $\mu$.

We observed that the Bayes estimators of $M, p$, and $R\left(t_{0}\right)$ under MELF and SELF appear to be robust with respect to the correct choice of prior parameters a $(b)$ and a wrong choice of the prior parameters $b(a)$. This can be seen from Table 3. These conclusions are also valid in case of GELF and LLF, as observed in Tables 4, 5, and 6.

\section{Simulation Results}

In this section, we have generated 500 different random samples with $M=3, N=30, L=7, t_{0}=6, p=0.3$, $r=t=s=3, k=15$ from the distribution defined in (1) to justify the results obtained in Section 8 and obtained the Bayes estimators and their risk $=E_{d}[L(d, \theta)]$ under SELF,
GELF, LLF and MELF for different choices of $(a, b)$. The results are summarized in Tables $7,8,9$, and 10 .

\section{Conclusions}

From Table 7, we observed that the Bayes estimators of the parameter perform well (minimum Bayes risk) under MELF compared to SELF for any choice of hyper parameters $a$ and $b$.

From Tables 8-10, we find that under both the asymmetric loss functions, namely, GELF and LLF, Bayes risks become smaller when parameters $\left(\alpha_{1}\right.$ and $\left.\alpha_{2}\right)$ tend to zero, that is, Bayes estimators perform well under these asymmetric loss functions.

From Tables 7-10 following Calabria and Pulcini [13], we also observed that the Bayes estimators of the parameters are robust with respect to the correct choice of prior parameters $a(b)$ and a wrong choice of the prior parameters $b(a)$ which we have concluded in Section 8.

\section{References}

[1] M. Yaqub and A. H. Khan, "Geometric failure law in life testing," International Journal of Pure and Applied Mathematical Sciences, vol. 14, no. 1-2, pp. 69-76, 1981. 
[2] M. N. Patel and A. V. Gajjar, "Progressively censored samples from geometric distribution," The Aligarh Journal of Statistics, vol. 10, pp. 1-8, 1990.

[3] N. W. Patel and M. N. Patel, "Some probabilistic properties of geometric distribution," International Journal of Management and System, vol. 22, pp. 1-3, 2006.

[4] N. Balakrishnan, "On the maximum likelihood estimation of the location and scale parameters of exponential distribution based on multiply type II censored samples," Journal of Applied Statistics, vol. 17, no. 1, pp. 55-61, 1990.

[5] H. Fei and F. Kong, "Internal estimations for one and two parameter exponential distributions under multiple type-II censoring," Communications in Statistics-Theory and Methods, vol. 23, no. 6, pp. 1717-1733, 1994.

[6] F. Kong and H. Fei, "Limit theorems for the maximum likelihood estimate under general multiply type II censoring," Annals of the Institute of Statistical Mathematics, vol. 48, no. 4, pp. 731-755, 1996.

[7] L. D. Broemeling and H. Tsurumi, Econometrics and Structural Change, Marcel Dekker, New York, NY, USA, 1987.

[8] I. Guttman, Statistical Tolerance Region: Classical and Bayesian, Griffin, London, UK, 1970.

[9] V. M. Rao Tummala and P. T. Sathe, "Minimum expected loss estimators of reliability and parameters of certain lifetime distributions," IEEE Transactions on Reliability, vol. 27, no. 4, pp. 283-285, 1978.

[10] R. Calabria and G. Pulcini, "An engineering approach to Bayes estimation for the Weibull distribution," Microelectronics Reliability, vol. 34, no. 5, pp. 789-802, 1994.

[11] H. R. Varian, "A Bayesian approach to real estate assessment," in Studies in Bayesian Econometrics and Statistics in Honor of Leonard J. Savage, Stephen E. Fienberg and A. Zellner, Eds., pp. 195-208, North-Holland Publishing Company, Amsterdam, The Netherlands, 1975.

[12] I. S. Gradshteyn and I. M. Ryzhik, Table of Integrals, Series and Products, Academic Press, Salt Lake City, Utah, USA, 2000.

[13] R. Calabria and G. Pulcini, "Point estimation under asymmetric loss functions for left-truncated exponential samples," Communications in Statistics-Theory and Methods, vol. 25, no. 3, pp. 585-600, 1996. 

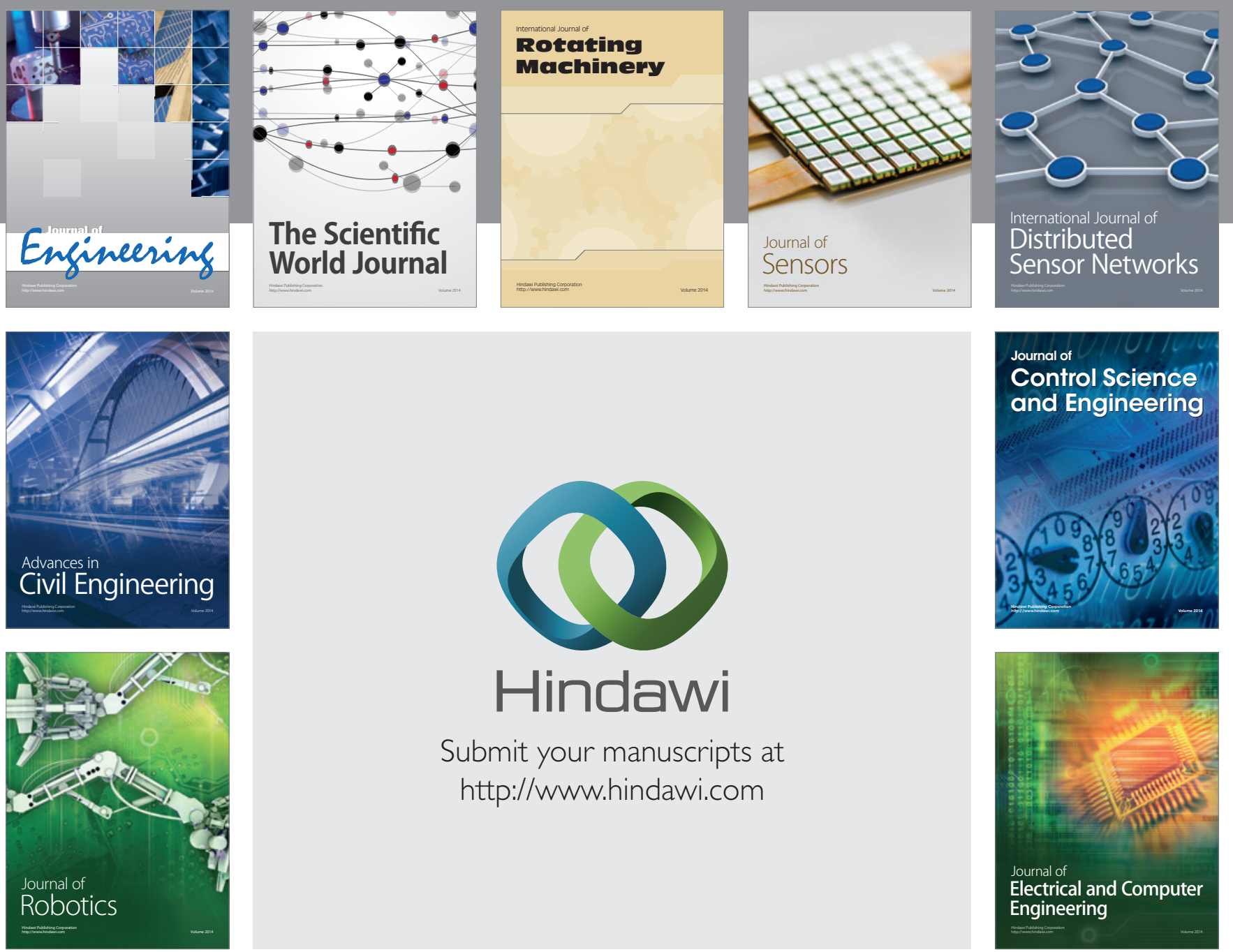

Submit your manuscripts at

http://www.hindawi.com
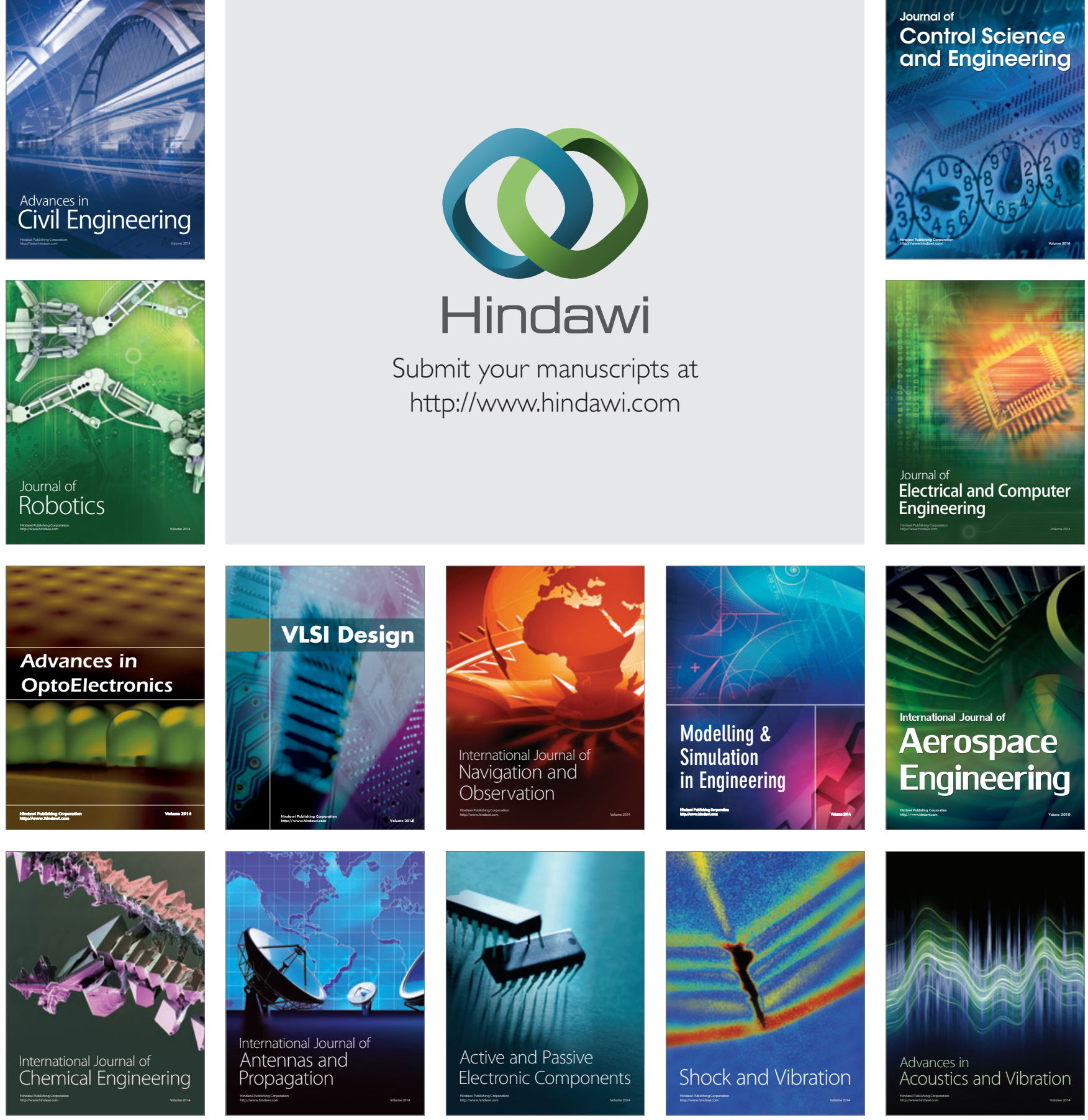